\title{
NOTICIAS
}

\section{¿Hacia dónde va A mérica Latina?, reflexión en el Congreso Latinoamericano de Sociología}

En el marco de los procesos socioeconómicos, políticos y culturales que definen la entrada al nuevo milenio, la Asociación Latinoamericana de Sociología (ALAS) propuso un encuentro analítico-reflexivo los días 12 al 16 de octubre de 1999, en Santiago de Chile, cuyo lema fue: "¿Hacia dónde va América Latina?"

La respuesta a la iniciativa fue variada en la asistencia, crítica en las perspectivas y diversa en las propuestas; éstas últimas fueron hechas desde el punto de vista de distintas disciplinas como la sociología, la psicología, la antropología, la economía, la medicina, la ecología y, en general, desde la perspectiva de investigadores y científicos sociales. La presencia de México y demás países latinoamericanos con trabajos de investigación, aportó nuevos enfoques para explicar la situación de la investigación sociológica en cada una de las naciones y, en general, de América Latina.

El evento fue organizado en mesas de trabajo, y los temas que abordaron algunas de ellas fueron: restructuración económica internacional, globalización y estados nacionales; reforma del Estado, políticas públicas y sociales; sistemas políticos y transición democrática; universidad y sociedad; sistemas de salud y seguridad social; dinámica demográfica y cambio social, y metodologías de la investigación social.

En esta última mesa, se discutió de manera crítica el uso de diferentes herramientas cuantitativas y cualitativas en los diversos ámbitos profesionales; cabe destacar que recurrentemente se señalaba a la salud como uno de los campos donde mejor se refleja el beneficio de esta convergencia metodológica. En los últimos años, la investigación en salud en diferentes países como Argentina, Bolivia, Venezuela, Chile y México, ha mostrado ser un espacio en el que se van articulando de mejor manera las decisiones teóricas y las elecciones prácticas.

Se habló del tiempo y el espacio como variables intrínsecas al análisis social, ya que permiten captar el movimiento y la diacronía de las realidades sociales.

Se señaló que es preciso trascender la aparente contradicción que existe entre la investigación cualitativa y la cuantitativa, y se subrayó la necesidad de fortalecer aquellas estrategias de investigación que escapen a dicha contienda.

La información proporcionada permitió obtener una imagen integral de la forma en que están estruc- turadas nuestras sociedades en el marco del fin del milenio.

Dra. Blanca Pelcastre Villafuerte, M.S.P.Verónica De León Reyes. Centro de Investigación en Sistemas de Salud, Instituto Nacional de Salud Pública, M éxico.

\section{Reunión Anual de la A sociación Norteamericana de Evaluación (AEA) "The territory ahead: Foundations and frontiers". Orlando, Florida. 2-6 de noviembre de 1999}

Esta es la cuarta reunión anual de la Asociación de Evaluadores, que en los últimos cuatro años ha creado un foro de intercambio entre sus miembros, y ha dado visibilidad, entre las agencias intergubernamentales y las fundaciones, a este grupo multidisciplinario de académicos que profesionalmente se han desarrollado en la aplicación de métodos de investigación científica a la evaluación de proyectos de desarrollo social, de salud, y de tecnología de servicios, colegiado de profesionistas. La AEA registra 700 profesionistas dedicados de tiempo completo a la evaluación, lo cual parece indicar la emergencia de una entidad profesional nueva. El presidente saliente de la AEA, Mi- 
chael Scriven, doctorado en matemáticas y en psicología, dijo que esta profesión requiere de maestría en varias operaciones complejas que incluyen diseñar investigación evualuativa, documentar hechos mediante la observación, analizar información secundaria, establecer el valor relativo en una escala, priorizar, atribuir efectos y predecir, además de capacidad para identificar y resolver conflictos grupales, institucionales y hasta de índole político. Todo ello con un fin práctico: proporcionar evidencia sobre "cómo servir mejor a la población del mundo".

La AEA se organiza en su interior a en grupos de interés temático (Topical Interest Group TIGs) que participan en redes de comunicación electrónica, entre ellas la red EVALTALK a la que accesan todos los agremiados. Quizá el beneficio más importante de la membresía es uno de los menos tangibles: un fuerte sentido de comunidad, de intercambio académico que caracteriza la mística de esta joven asociación. La trascendencia de la evaluación profesional en nuestros días, afecta áreas como el empoderamiento de los consumidores con información validada, como la expansión de una cultura que considera estándares, indicadores y parámetros en las decisiones individuales, comunitarias e institucionales, como un factor de influencia en las políticas no sólo de asistencia social sino hasta en la asignación graduada de impuestos según capacidad de contribución.

La reunión AEA 99 se constituyó de más de 100 sesiones, incluyendo paneles patrocinados por agencias gubernamentales como The National Science Foundation, e intergubernamentales como el Banco Mundial, y por Fundaciones como la WK Kellogg, y la Robert Wood John- son, Care. Participaron más de 400 asociados, la mayoría académicos de más de 60 universidades de EUA, Canadá, Inglaterra, Suiza y América Latina, entre las cuales destacan Standford, Chapel Hill, Illinois, Harvard, McMaster, y centros de investigación, como el Research Triangle y The Evaluation Forum, otros. Además, en el día previo y el subsiguiente a la Reunión se ofrecieron 14 talleres de desarrollo profesional $\mathrm{Di}$ seño de investigación evaluativa, El análisis estadístico no paramétrico, análisis cualitativos, filosofía y teoría de la evaluación, desarrollo de habilidades de investigación, meta evaluación, distribución geográfica y mapeo de información evaluación multicéntrica y de conglomerados, género y evaluación para el empoderamiento.

Es evidente, en los contenidos del programa de la Reunión, el interés de las agencias intergubernamentales por promover entre instituciones, grupos y comunidades de países en vías de desarrollo, la capacidad de evaluación, y su énfasis en incluir profesionistas provenientes de países apoyados por estas agencias y fundaciones. Un panel "Construyendo capacidad de evaluación en países en desarrollo" lo integraron especialistas en evaluación del Banco Mundial; así como "Diálogo sobre evaluación en los países en desarrollo", "Asociación para evaluación en centros internacionales" con la participación de la Universidad de Western Michigan, la Universidad de Peking, la de Minnesota y la de Otawa. Se destacó la participación de ponentes de Brasil (Instituto Ayrton Senna), de México (Instituto Nacional de Salud Pública, Universidad de Chapingo, Centro de Investigaciones Regionales Dr. Noguchi), la Universidad de Cos- ta Rica y Care de Perú, entre otras. Para mayor información consúltese la página de la AEA: www.eval. org.

Dra. Rosa María Núñez Urquiza. Jefa del Departamento de Sociedad, Familia y Salud.

Centro de Investigación en Sistemas de Salud, Instituto Nacional de Salud Pública

\section{Conclusiones de la conferencia sobre las reformas de salud en A mérica Latina}

Las experiencias de reforma en otras partes del mundo no han generado ni generarán una fórmula mágica para los gobiernos de América Latina. Sin embargo, sí pueden proporcionar lineamientos útiles que deberán adaptarse a las condiciones específicas de cada país.

Esta es la conclusión más general de la conferencia La Reforma de los Sistemas de Salud en América Latina: Aprendiendo de los Éxitos y los Fracasos de Otros Países, organizada por Latin American Newsletters y la Escuela de Economía de la Universidad de Londres, y llevada a cabo los días 16 y 17 de septiembre en la ciudad de Londres, Inglaterra el año próximo pasado.

Las nueve conclusiones fundamentales de este evento fueron:

1. La seguridad social y las fuerzas del mercado se deben considerar como herramientas para alcanzar el objetivo de la cobertura universal de los servicios de salud.

2. La segmentación social y geográfica produce sistemas de salud ineficientes. 
3. Independientemente de que se recurra a proveedores de salud públicos o privados, el sistema ideal es aquel que los integra en un solo marco.

4. El sistema financiero debe centralizarse.

5. Los estándares deben ser diseñados por los gobiernos nacionales.

6. Pacientes y médicos deben contar con libertad para elegir al proveedor ideal para cada situación.

7. Se requiere de un sector público más fuerte.

8. Debe evitarse a toda costa la discriminación de riesgos.

9. Se requiere de un mayor financiamiento público.

Cabe destacar que el doctor Julio Frenk, director ejecutivo de Pruebas Científicas e Información para
Políticas, de la Organización Mundial de la Salud, dictó la primera conferencia latinoamericana escuchada en los eventos patrocinados por Latin American Newsletter. El título de su ponencia fue "Hacia un nuevo universalismo: una visión de políticas de salud en América Latina". 